\title{
Research on Scientific Collaboration Social Network of Provincial Academy of Agricultural Sciences
}

\author{
Xia-ling $\mathrm{CHU}^{1, a}$, Xiu-jie HUANG ${ }^{2, b, *}$, Hui-ling ZHANG ${ }^{3, c}$, \\ Xue-na BAI ${ }^{4, d}$, Jian-Xun $\mathrm{CUI}^{5, \mathrm{e}}$, Xian-zhi YANG ${ }^{6, \mathrm{f}}$ and \\ Yi-guang $\mathrm{ZOU}^{7, g}$
}

1,2,3,4,5,6,7 Institute of Agricultural Economics and Rural Development, Guangdong

Academy of Agricultural Sciences, Guangzhou, Guangdong 510640, China),

No. 31, Wushn Road, Tianhe District City, Guangzhou Province, Guangdong

$$
\begin{aligned}
& \text { Province, China } \\
& \text { a158283003@qq.com, b124851033@qq.com, c171695452@qq.com, } \\
& \text { dbaixuena1983@163.com, e15392925@qq.com, f13662312531@163.com, } \\
& \text { 940711626@qq.com }
\end{aligned}
$$

Keywords: Provincial Academy of Agricultural Sciences, Social network analysis, Co-authorship social network, Scale-free network.

\begin{abstract}
A research was conducted on the co-authorship social network of Guangdong Academy of Agricultural Sciences by using bibliometrics and social network analysis (SNA), then statistics and analysis were conducted on the three statistic characteristics of scale-free network: average network path length, node degree distribution and condensed subsets. Results showed that Guangdong Academy of Agricultural Sciences had 3746 co-authorship network nodes and 9307 connection lines. The average network path was 12.81183 and network density was 0.00158670 . For the total 3746 network nodes, the average degree was 2.96796583 , among which the maximum was 80 and the minimum was 1 . The connection distribution of collaboration network was uneven; a large number of nodes had few connections while a few nodes had most of connections in the network. The network was distributed to 597 condensed subsets, among which the largest condensed subset had 2156 nodes, accounting for $57.555 \%$ of the total nodes. There were 24 medium-sized subsets and the number of nodes was about 6 to 22. The co-authorship network was incompletely connected and the integral connection was fairly close. There were some closely connected groups in large sub-network and the integral co-authorship status was perfect. Different nodes of the co-authorship network were connected closely, among which the top 50 authors collaborated with other scientific research personnel frequently. Such kind of organization form of Guangdong Academy of Agricultural Sciences yet has great advantage in key agricultural science breakthroughs. It is reflected in those aspects: the forming mechanism of large-scale co-authorship network has close connection with the stability and durability of talents in this field, the breakthrough of key scientific projects can also lead to the formation of large-scale co-authorship network.
\end{abstract}

Author: Xia-ling CHU (1985-), female, master, Assistant research fellow, E-mail: 158283003@qq.com, Tel.18988920513

Corresponding author: Xiu-jie HUANG(1980-), male, master, associate research fellow, E-mail: 124851033@qq.com 


\section{Introduction}

With the development and specialization of science, researchers need to collaborate to accomplish many scientific researches. On academy, it is reflected in frequent scientific research collaboration and science co-authorship. Researchers engaged in scientific research collaboration and science co-authorship are usually familiar with each other and collaborate to publish papers and establish a co-authorship publication network. This network can truly reflect the researchers' human network, also known as social network. Collaboration social network of scientific field is a complex scientific research collaboration network composed of different participants and they have extensive connection. In this network, network nodes represent researchers in scientific fields and network connection represents the relation of papers co-published by the researchers. The structure of collaboration social network of scientific field reflectes the network variables such as how the participants connect, connection degree and node distribution etc, which will influence the collaboration degree of researchers of scientific fields in the network and the development trend of network directly. In the past, bibliometrics were used to analyze the co-authorship relation. The disadvantage of such analysis was that the co-authorship relation was not explored as a whole and the underlying relation of some authors was ignored. Social network analysis was derived from sociology and has been developed as one of widely-used research methods nowadays. By using social network analysis (SNA) to explore the collaboration relation among authors, we can know the co-authorship status of some certain fields and scientific research range for strengthening collaboration.

Many foreign scholars have conducted empirical study on co-authorship network, however, such researches mainly focused on the natural sciences and authors usually collaborated in these fields. Scholars like Fuyuki Yoshikane made a comparison of co-authorship network between the filed of computer science research and the field of application research and modified the hits-algorithm to identify leaders and followers of the co-authorship network. Barabasi A L conducted a study on the evolution rules (with time variation) of co-authorship network and verified that: co-authorship network distribution was compliance with power law distribution, the clustering coefficient and average degree declined with time, proportion of maximally connected sub-graph of the whole network increased with time, new nodes preferred to establish connection with higher nodes. New-man M E J conducted a study on the inherence of co-authorship network in the fields of physics, biology and computer science and made a comparison and analysis of different networks. Scholars like Yasmin H made an introduction of the basic methods of social network analysis such as centrality analysis and cluster analysis and his research focused on the small group features formed by the cluster analysis of co-authorship network, which can be summarized as single type, consultant, enterprise and team and speculated the impacts of these small group features. In domestic, more researches were conducted to make empirical analysis on co-authorship network by SNA. Qinghua Zhu made a summary on the application status of SNA in the field of information science. He made an empirical analysis on the co-authorship social network of Journal of the China Society For Scientific and Technical Information, conducted the centrality analysis, cohesive subgroup analysis and core-remote structure analysis, evaluated the scholars' position in the co-authorship network, found the closely connected groups and figured out the number and organizations of the core authors in the co-authorship network. Bei Liu conducted a study on researchers' co-published network, co-word network, citation network and collaboration network of small groups in different periods. Aixian Jiang 
conducted a research on the collaboration network of management science. However, majorities of researches were conducted to analyze co-authorship social network of some certain science and certain magazines, but less study was conducted on that of a certain organization.

Provincial academy of agricultural sciences plays an important role in scientific agricultural research in China. By coordinating the agricultural science and technology resources of the whole province and gathering the talents to strengthen regional collaborative innovation and development, the provincial agricultural research leader head the development of agricultural research of the province. Meanwhile, there are many research institutes in provincial academy of agricultural sciences, mainly including institutes of grain crops, vegetables, cash crops, plant protection, soil and fertilizer, animal husbandry and veterinary and agricultural economy etc. The integration of departments will help to integrate the interacting factors of those departments and gather them into a same task to achieve the effects of collaborative innovation that single innovation can not reach. In the meantime, provincial academy of agricultural sciences has research collaboration with office for agricultural research at local levels to extend the coverage of agricultural research in the whole province and puts agricultural research closer to frontline of agricultural production. However, no research on co-authorship network of specific research group has been conducted in domestic or abroad. Only Huili Wang conducted a study on the collaboration relation of professors of biological medicine of one provincial medical university. And Shanmin Ding conducted a research on the co-authorship relation of Institute of Chemistry of Tianjin Normal University. Therefore, it is necessary to make an analysis on the research collaboration network of provincial academy of agricultural sciences from the perspectives of network individuality attribute and integrity attribute to discover its connotation.

\section{Materials and Methods}

\section{Data Collection}

The scientific paper data including the papers and authors' information of Guangdong Academy of Agricultural Sciences from 2006 to 2015 were obtained through online search of China National Knowledge Infrastructure(CNKI), Wanfang Database, VIP database etc. by NoteExpress Software. A database was further established based on the collective data which were classified as thesis title, author, institution, publication date, journal and keywords, and such data were stored and handle by Excel.

\section{Data Cleaning}

There were some replication and inaccuracy in the raw data obtained by software, and such data would cause errors in the later analysis results. Therefore, it is necessary to clean the data. Softwares like NoteExpress and Excel were used to clean the data, and 5885 groups of basic data were obtained.

On the process of data cleaning, one of the key steps is to distinguish and deal with the duplicated authors. Considering the actual need of network analysis, we can reduce the statistical errors caused by duplicated names if we follow the methods below:

(1) Combine the author' name and organization to identify whether the synonymous author matches with the same person. 
(2) There are different ways of expression on the organizations of some authors, it is needed to select and compare the key information of the organization to further identify synonymous author.

(3) One author may publish papers in various periods at different stages. The author at different stages may be identified as different ones if we put much emphasis on the combination of name and organization, which will also cause many problems during the process of statistics and analysis.

(4) In order to reduce the errors brought by the above mentioned methods, co-authorship network can be established to verify and revise the data. In the network, the shortest path of two synonymous authors with different institutions is 2 , which represents that they ever collaborated with each other and can be considered as the same person. Repeatedly, some over-distinguished synonymous authors can be revised to improve the accuracy of the data.

\section{Establishing Network}

As the research focused on the co-authorship connection between authors, only related data of co-authors' were selected to establish co-authorship network. Totally, 5559 groups of effective data were selected. As the collected raw data were recorded in the form of "one paper to several authors", while the establishment of network was connected by taking authors as nodes. Such form needed to be transformed to the form of that authors collaborate in pairs in Excel table. Data stored in Excel table could not be entered into Pajek software and needed to be transformed into corresponding ".net" form through Excel2pajek software.

\section{Results and Analysis}

\section{Network Characteristic Parameter}

Average Path Length. Average path length refers to the average length of two random nodes. As the co-authorship network of provincial academy of agricultural sciences was an incompletely connected network and the average path length would be infinitely large if following general statistics. In order to avoid such situation, node pairs with no connected path would not be taken into account. However, the co-author network of provincial academy of agricultural sciences was usually part of agricultural research field and different nodes may have connections. Therefore, all nodes were analyzed in this research. Results showed that the longest path of co-authorship network of Guangdong Academy of Agricultural Sciences was 40 and the corresponding node was Xiaoman She and Xiaoqing Xue, which meant that these two people could be connected through 40 inter-linkages. For the whole network, there were 3746 nodes and the number of connection was up to 9307 (Figure1).

The average network path was 12.81183 and it meant that two random authors could set up co-authorship connection through a path of 12.81183 at the most. Compared to that of a certain subject or a certain college, the path of this network was longer. There were 15 research institutes (such as Rice Research Institute, Vegetable Research Institute etc.) under Guangdong Academy Agricultural of Sciences. The network scale became larger with the emergence of new subjects and engagement of new researchers, which further led to longer path. 


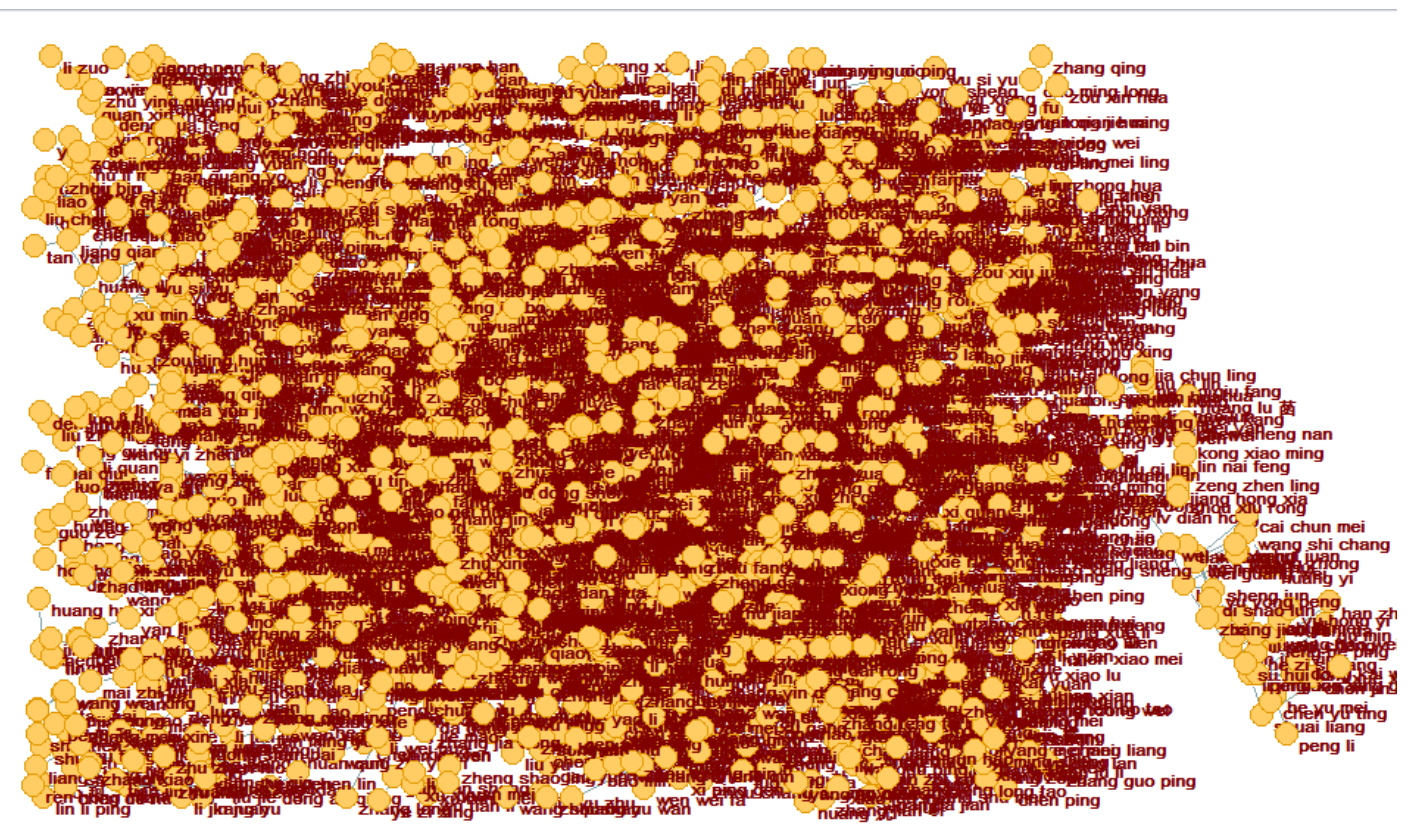

Figure 1. Co-authorship network of research personnel of Guangdong Academy Agricultural of Sciences

Network Density. Calculated by Pajek Software, the co-authorship network density of Guangdong Academy of Agricultural Sciences was 0.00158670. The network density was much lower compared to the largest density value (0.5) of actual network. Considering the larger scale of actual network involving collaboration connection of different organizations, the density was higher compared to ever-researched data (Mengwei 0.0002323), which showed that the network density of Guangdong Academy of Agricultural Sciences was higher and the collaboration of nodes was relatively close. In actual network, a person has limited ability and time to maintain certain relations and the person will stop establishing new relation if he gives more and gains less, especially for large-scale network.

Degree Distribution. In undirected network, degree describes the number of edges connected to specified nodes and this number reflects the importance of this node from one certain respect and is one of canonical measure of node centrality. The results showed that for the 3746 nodes in the whole network, the average degree of co-authorship network of Guangdong Academy of Agricultural Sciences was 2.9679658 (Maximum: 80, minimum: 1). From figure 2, it was showed that the connection distribution of the collaboration network was uneven, a large number of nodes had few connections, while a few nodes had most of connections in the network.

Analysis on Condensed Subsets. If classified based on condensed subset, the network was distributed to 597 condensed subsets (Figure 3), among which the largest condensed subset has 2156 nodes, accounting for $57.555 \%$ of the total nodes. There are 24 medium-sized subsets and the number of nodes was about 6 to 22 (Figure 4).

Results showed that the co-authorship network was incompletely connected and the integral connection was fairly close. There were some closely connected groups in large sub-network and the integral co-authorship status was perfect. Results (Figure 4) showed that the medium-sized condensed subsets had the nodes representing the staff ever worked for Guangdong Academy of Agricultural Sciences and they still collaborated closely with the Academy after they left the original organizations. In addition, there were 572 small-sized networks in the co-authorship network and most of them were composted of 3 to 5 nodes. The formation of such small-sized networks 
was mainly because: First, the research area was special and small stable groups were formed. The core node of the small-sized network was Yu Chen and he was engaged in the research of rice genomics with Fen Chen, Jiangping Song and Dujuan Li. The research field was self-contained and the research group was relatively stable. Second, the research members worked for several organizations and Guangdong Academy of Agricultural Sciences was one of the organizations they worked for. As shown in Figure 7, the group of Huilong Su was not only the staff of Veterinary Research Institute of Guangdong Academy of Agricultural Sciences but also the staff of Veterinary College of South China Agricultural University. Third, some new administrative personnel needed to publish papers based on professional title and did not establish close connection with other research personnel.

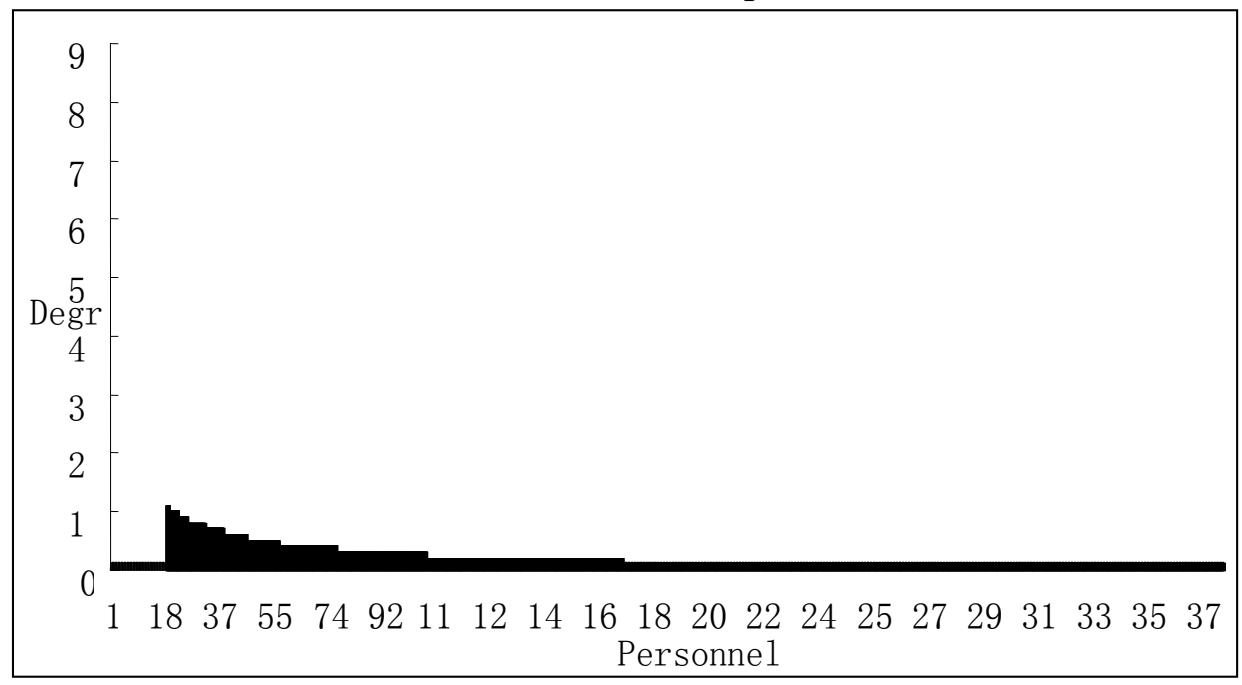

Figure 2. Distribution of nodes

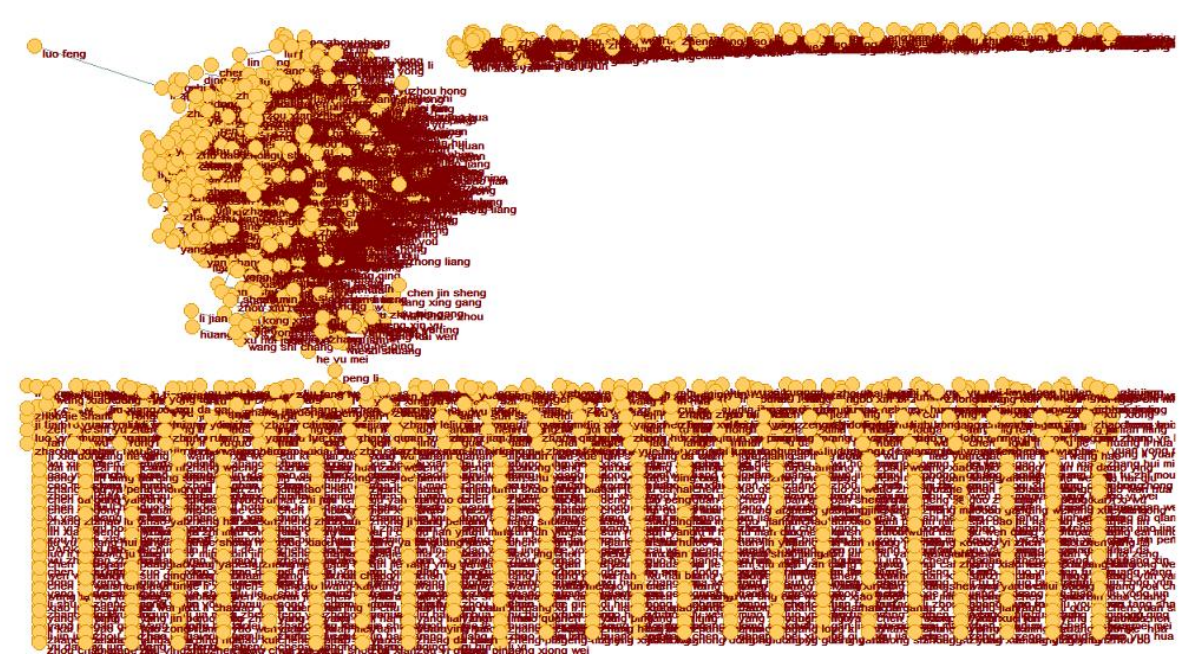

Figure 3. Distribution of co-authorship network of condensed subsets of Guangdong Academy of Agricultural Sciences 


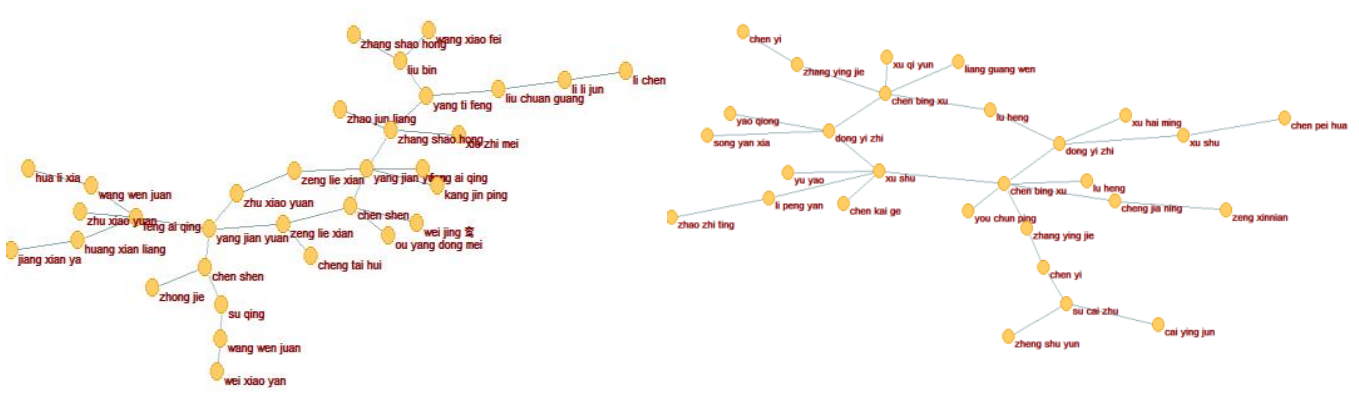

Figure 4. Part of medium-sized condensed subsets

\section{Analysis on Authors' Effects}

The centrality of certain nodes in the network reflects the position and influence of corresponding individuals in the network. Therefore, the analysis of the influence of authors can be converted to the analysis of node centrality of co-authorship network accordingly. The advantages and disadvantages of the co-authorship network could be found to provide experience for improving research collaboration in the future.

Results (Table 1) showed that different nodes of the co-authorship network of Guangdong Academy of Agricultural Sciences were connected closely, among which the top 50 authors collaborated with other scientific research personnel frequently. From the analysis of the authors' identities, it was found that the top 50 authors were usually the leader of the subject and led a corresponding subject team. From the perspective of centrality (Table 2), centrality of nodes ranking top 50 played an important role in the co-authorship collaboration network and the collaboration of whole research network would be highly dependent on those nodes. Compared to the top 50 authors, the authors with centrality ranking top 50 played a different role in a team. Some authors were still the leader of corresponding subject, such as Taisen Liao. Some authors conducted research of wide fields and connected the whole network, like Xia Feng and Junrong Qiu etc. Some authors connected the whole network because of subject area, such as the incumbent or former members of Institute of Agricultural Economics and Rural Development: Yelu Zheng, Canfang Zhou, Xiujie Huang, Zhong Wan, Haowen Luo, Jianjun Hong, Wei Fang etc. From the research results of centrality, if one author has collaborated with the authors in several cohesive subsets and he would function as a bridge that connected these subgroups. The shortest path of other authors in these subgroups would inevitably connect this author (if there was only one author functioned as a bridge between these subgroups), the degree of this node became higher accordingly. 
Table1. Authors of Top 50 ranking individual degree value of co-authorship network of Guangdong Academy of Agricultural Sciences

\begin{tabular}{|c|c|c|c|}
\hline Ranking & Intensity & Author & Title/Position \\
\hline 1 & 80 & Jun-ming CAO & Deputy secretary/Researcher \\
\hline 2 & 75 & Sen-tai LIAO & Secretary/Researcher \\
\hline 3 & 67 & Geng-sheng XIAO & Deputy dean/Researcher \\
\hline 4 & 64 & Ming-wei ZHANG & Director/Researcher \\
\hline 5 & 55 & Yu-juan XU & Deputy Director/Researcher \\
\hline 6 & 51 & Zong-yong JIANG & Former Dean/Researcher \\
\hline 7 & 46 & Zhong WAN & Director/Researcher \\
\hline 8 & 42 & Can-fang ZHOU & Director/Research Associate \\
\hline 9 & 38 & Pei-zhi XU & Deputy Director/Researcher \\
\hline 9 & 38 & Gan-jun YI & Deputy Dean/Researcher \\
\hline 11 & 37 & Ye-lu ZHENG & Former Director/Researcher \\
\hline 12 & 35 & Shao-chuan ZHOU & Director/Researcher \\
\hline 13 & 34 & Li-xian YAO & Researcher \\
\hline 13 & 34 & Chun-ling LI & Director/Researcher \\
\hline 13 & 34 & Chuan-guo LI & Director/Researcher \\
\hline 16 & 31 & Xue-ming LIU & Director/Researcher \\
\hline 17 & 28 & Zhi-ping PENG & Director/Researcher \\
\hline 17 & 28 & Shao-ying AI & Deputy Director/Researcher \\
\hline 19 & 27 & Li FENG & Director/Researcher \\
\hline 19 & 27 & Ji-wu ZENG & Deputy Director/Researcher \\
\hline 21 & 26 & Dong CHEN & Former Deputy Dean/Researcher \\
\hline 22 & 25 & Xiao-ming HE & Deputy Director/Researcher \\
\hline 22 & 25 & Jian-guang HU & Director/Researcher \\
\hline 22 & 25 & Hao-wen LUO & Director/Researcher \\
\hline 22 & 25 & Ying-an ZANG & Professor \\
\hline 26 & 24 & Han-cai CHEN & Director/Researcher \\
\hline 27 & 23 & Lu-xiang ZHAN & Director/Researcher Associate \\
\hline 27 & 23 & Yan ZHANG & Deputy Researcher \\
\hline 27 & 23 & Ai-tian PENG & Director/Researcher \\
\hline 27 & 23 & Shi-li SUN & Deputy Director/Researcher Associate \\
\hline 27 & 23 & Shuan-hu TANG & Director/Researcher \\
\hline 27 & 23 & Gen-fa ZHU & Deputy Director/Researcher \\
\hline 33 & 22 & Xiao-fang LI & Former Deputy Director/Researcher \\
\hline 33 & 22 & Wei-jun LIN & Deputy Director/Researcher \\
\hline 33 & 22 & Gui-hua LI & Associate Researcher \\
\hline 36 & 21 & Bao-mei YANG & Research Assistant \\
\hline 36 & 21 & Xing-hong HUANG & Director/Researcher Associate \\
\hline 36 & 21 & Qing HUANG & Director/Researcher \\
\hline 36 & 21 & Li-hua LV & Team Leader/Researcher \\
\hline 36 & 21 & Yong-hong HUANG & Researcher \\
\hline 41 & 20 & Xin-hua XIE & Researcher \\
\hline 41 & 20 & Zhi-qiang LI & Researcher Associate \\
\hline 41 & 20 & Bi-run LIN & Director/Researcher \\
\hline 41 & 20 & Ying-cai LIN & Deputy Director/Researcher \\
\hline 41 & 20 & Da-jian PAN & Director/Researcher \\
\hline 41 & 20 & Pei-yuan YUAN & Deputy Director/Researcher \\
\hline 47 & 19 & Da-sen XIE & Director/Researcher \\
\hline 47 & 19 & Shi-zi KUANG & Senior Agronomist \\
\hline 47 & 19 & Guo-liang LI & Research Assistant \\
\hline 47 & 19 & Kai-zhi XIE & Researcher Associate \\
\hline 47 & 19 & Fa-yuan DING & Researcher Associate \\
\hline 47 & 19 & Guo-qing LUO & Secretary/Researcher \\
\hline 47 & 19 & Zi-fu HE & Secretary/Researcher \\
\hline 47 & 19 & Dao-bang TANG & Researcher Associate \\
\hline 47 & 19 & Yuan-shan YU & Research Assistant \\
\hline
\end{tabular}


Table 2. Authors of Top 50 Ranking Individual Centrality of Co-authorship Network of Guangdong Academy of Agricultural Sciences

\begin{tabular}{|c|c|c|c|}
\hline Ranking & Centrality Value & Author & Title/Position \\
\hline 1 & 0.083341912 & Ye-lu ZHENG & Former Director/Researcher \\
\hline 2 & 0.080564024 & Can-fang ZHOU & Director/Researcher Associate \\
\hline 3 & 0.0722431130 & Sen-tai LIAO & Secretary/Researcher \\
\hline 4 & 0.0644594530 & Zong-yong JIANG & Former Dean/Researcher \\
\hline 5 & 0.0539313100 & Ting RONG & Research Assistant \\
\hline 6 & 0.0539217640 & Bing-zhi HUANG & Director/Researcher \\
\hline 7 & 0.0533251920 & Xiao-lang TANG & Researcher \\
\hline 8 & 0.0469808530 & Geng-sheng XIAO & Deputy Dean/Researcher \\
\hline 9 & 0.0458913110 & Zhi-qiang LI & Researcher Associate \\
\hline 10 & 0.0454178660 & Xiu-jie HUANG & Director/Researcher Associate \\
\hline 11 & 0.0435778790 & Pei-yuan YUAN & Deputy Director/Researcher \\
\hline 12 & 0.0412373490 & Dong CHEN & Former Deputy Dean/Researcher \\
\hline 13 & 0.0372351330 & Jun LIU & Director/Researcher \\
\hline 14 & 0.0333968080 & Zhuang CHEN & Form Deputy Director/Researcher \\
\hline 15 & 0.0330784630 & Zhong WAN & Dean/Researcher \\
\hline 16 & 0.0320723430 & Qiu-shuang WANG & Research Assistant \\
\hline 17 & 0.0307430790 & Zhi-hong XU & Director/Researcher \\
\hline 18 & 0.0291020380 & Zong-hua QIN & Research Assistant \\
\hline 19 & 0.0290600290 & Jun-xi CAO & Director/Researcher \\
\hline 20 & 0.0262476340 & Lu-xiang ZHANG & Director/Researcher Associate \\
\hline 21 & 0.0247090890 & Jun-ming CAO & Deputy secretary/Researcher \\
\hline 22 & 0.0243164020 & Gui-hua LI & Researcher Associate \\
\hline 23 & 0.0241588430 & Shi-li SUN & Deputy Director/Researcher Associate \\
\hline 24 & 0.0227977590 & Wei-jun LIN & Deputy Director/Researcher \\
\hline 25 & 0.0226466000 & Li-hua LV & Researcher \\
\hline 26 & 0.0210680490 & Jun-rong QIU & Deputy Director/Researcher \\
\hline 27 & 0.0204933800 & Wei-ming ZHANG & Director/Researcher \\
\hline 28 & 0.0196495220 & Cai-jin LING & Director/Researcher \\
\hline 29 & 0.0187760610 & Dong-mei LI & Research Assistant \\
\hline 30 & 0.0182200200 & Jian-feng YUAN & Research Assistant \\
\hline 31 & 0.0177483210 & Yu-juan XUAN & Deputy Director/Researcher \\
\hline 32 & 0.0172363890 & Hua-ling WU & Director/Researcher Associate \\
\hline 33 & 0.0172041350 & Ying-an ZANG & Professor \\
\hline 34 & 0.0156173010 & Xuan-qiang LIANG & Director/Researcher \\
\hline 35 & 0.0154057220 & Ji-shui QIU & Director/Researcher \\
\hline 36 & 0.0153589970 & Li-xia MU & Research Assistant \\
\hline 37 & 0.0152279800 & Hao-wen LUO & Director/Researcher \\
\hline 38 & 0.0150110650 & Han-cai CHEN & Director/Researcher \\
\hline 39 & 0.0146090310 & Xiao-ming HE & Deputy Director/Researcher \\
\hline 40 & 0.0137874290 & Gen-fa ZHU & Deputy Director/Researcher \\
\hline 41 & 0.0136551040 & Zhi-yong FENG & Director/Researcher \\
\hline 42 & 0.0134358790 & Dao-bang TANG & Researcher Associate \\
\hline 43 & 0.0132749050 & Yu-rong HE & Professor \\
\hline 44 & 0.0128554070 & Yue-rong WEI & Researcher Associate \\
\hline 45 & 0.0128420020 & Jian-jun HONG & Section Chief/Researcher \\
\hline 46 & 0.0127063500 & Shuan-hu TANG & Director/Researcher \\
\hline 47 & 0.0127024240 & Wei FANG & Director/Researcher Associate \\
\hline 48 & 0.0121551570 & Ying-jie MEI & Section Chief/Research Assistant \\
\hline 49 & 0.0119582090 & Xia FENG & Deputy Director/Researcher \\
\hline 50 & 0.0119337670 & Shao-hai YANG & Deputy Director/Researcher \\
\hline
\end{tabular}




\section{Conclusions}

The results showed that Guangdong Academy of Agricultural Sciences had 3746 co-authorship network nodes and 9307 connection lines. The average network path was 12.81183 and network density was 0.00158670 . For the total 3746 network nodes, the average degree was 2.96796583 , among which the maximum was 80 and the minimum was 1 . The connection distribution of collaboration network was uneven, a large number of nodes had little connection while a few nodes had most of connection in the network. If classified based on condensed subset, the network was distributed to 597 condensed subsets, among which the largest condensed subset had 2156 nodes, accounting for $57.555 \%$ of the total nodes. There were 24 medium-sized subsets and the number of nodes was about 6 to 22. The co-authorship network was incomplete connected and the integral connection was fairly close. There were some closely connected groups in large sub-network and the integral co-authorship status was perfect. In addition, there were 572 small-sized networks in the co-authorship network and most of them were composted of 3 to 5 nodes. Different nodes of the co-authorship network were connected closely, among which the top 50 authors collaborated with other scientific research personnel frequently. From the analysis of the authors' identities, it was found that the top 50 authors were usually the leader of the subject and led a corresponding subject team. From the perspective of intermediary, centrality of nodes ranking top 50 played an important role in the co-authorship collaboration network and the collaboration of the whole research network would be highly dependent on those nodes. Compared to the top 50 authors, the authors with centrality ranking top 50 played a different role in a team. Some authors were still the leader of corresponding subject. Some authors conducted research of wide fields and connected the whole network. Some authors connected the whole network because of subject area.

Results showed that such kind of organization form of Guangdong Academy of Agricultural Sciences yet had great advantages in key agricultural science breakthroughs. It was reflected in:

(1) The forming mechanism of large-scale co-authorship network had close connection with the stability and durability of talents in this field. From the research results, it was found that the research team composted of key authors such as Junming Cao, Sentai Liao, Zongyong Jiang, Gengsheng Xiao and Zong Wan etc., had great advantages in the scale of co-author network and the number of collaboration organizations. In the network, there were many core authors and bridge-oriented authors in the largest cohesive subgroups, which led to the close collaboration of the affiliated scientific research institutes and the small groups could be connected to large-scale co-authorship network.

(2) The breakthrough of key scientific projects could also lead to the formation of large-scale co-authorship network. For the breakthrough of major projects, the current national funding system played a leading role in scientific research collaboration. There were two types of academic team: The first type was problems-tackling academic team, which referred to the scientific research team formed to tackle major problems of national economy and social development. The second type was loose-collaboration academic team, which developed due to common interests or common views spontaneously. Per the research results, the largest cohesive subsets in the co-authorship network of Guangdong Academy of Agricultural Sciences could be regarded as problem-tackling team composted of major research groups of crops, soil 
and fertilizer, plant protection, genome and industrial economics and had natural advantages in the application and implementation of significant programs.

\section{Acknowledgment}

This research was financially supported by the projects: Construction of sharing platform and core competence of agricultural journals (Guangdong provincial science and technology planning project, 2013B060200009), Construction of agricultural technology transfer innovation service platform in Guangdong Province (Guangdong provincial science and technology planning project, 2014B040404062), Research on competitiveness promotion of Guangdong agricultural journals based on the evaluation of core journals (Dean fund project of Guangdong Academy of Agricultural Sciences, 201410).

\section{References}

[1] Yang ZHANG, Jin-yuan LIU, On the Co-authorship Network of Competitive Intelligence Field in China-Based on SNA, Documentation, Information and Knowledge. 2 (2012) 87-94. In Chinese.

[2] Ji-yang ZHANG, Ning LI, Analysis on Development of Scientific Co-authorship Network Research, Information studies:Theory \& Application. 4 (2012) 124-128. In Chinese.

[3] Wen-juan LI, Chunhua NIU, Empirical Study of the Use of Social Network Analysis in Analyzing the Co-Authorship Network--A Study on Journal of Library Science in China, Journal of Modern Information. 10 (2012) 153-158. In Chinese.

[4] Yu-lin CHEN, A Study of Construction of Mapping Knowledge Domain on Chinese Educational Technology Scholars' Coauthor Network, Journal of Distance Education. 6 (2012) 11-17. In Chinese.

[5] Liang LI, Qinghua ZHU, An Empirical Study of the Use of Social Network Analysis in Analyzing the Co-Authorship Network, Information Science. 4 (2008) 549-555. In Chinese.

[6] Wei MENG, Jin-gan PANG, Application of Pajek in the Visualization Research of Co-authorship Network of Information Science, Information studies: Theory \& Application, 4 (2008) 573-575. In Chinese.

[7] Wei MENG, Jin-gan PANG, Research on the Scientific Research Co-authorship Network of Information Science in China \& Analysis of Its Characteristic Parameters, Information studies: Theory \& Application. 8 (2009) 12-15. In Chinese.

[8] Kun DING, Ze-yuan LIU, Sheng-bo LIU, The Network Character and College Team Status in Coauthor Network of Science of Science and Management of Science and Technology, Science of Science and Management of S.\& T. 12 (2009) 10-15. In Chinese.

[9] Zhi-hong SONG, Yu-ying SHI, Dong-mei LI, The effects of academic paper quality on the network positions of stars in co-authorship network- the Case of "Innovation network" literature (1990-2012), Studies in Science of Science. 5 (2014) 660-668. In Chinese. 
[10] Wei CHEN, Wen ZHOU, Yi-fu LANG, Analysis of Scientific Collaboration Network Based on Co-author Network and Citation Network, Information studies: Theory \& Application. 10 (2014) 54-59. In Chinese.

[11]Li-juan ZHU, Jian-rong YU, Research of Weight Measurement of Co-author Networks Based on Author Contribution, Library Journal. 5 (2011) 16-20, 92. In Chinese.

[12]Xiao-hua YAO, An Empirical Study of Co- Authorship Network in LIS Institution Based on Social Network Analysis, Modern Information. 7 (2011) 51-54. In Chinese.

[13] Ji-min WANG, Ming-zi LI, Peng ZHANG, Co-authorship Network Analysis in the Research Field of Search Engine's Log Mining, New Technology of Library and Information Service. 4 (2011) 58-63. In Chinese.

[14]Feng-min YU, Jian-min TANG, A Study on Visualization Identification of Large-scale Co-authorship Network in the Field of Information Science[J]. Information studies: Theory \& Application, 8 (2011) 41-46. In Chinese.

[15] Qing-hua ZHU, Zhe FAN, Wen-wei SHI, Information studies: Theory \& Application, 11 (2011) 6-10, 20. In Chinese.

[16]Feng-ming YU, Jiang-ming TANG, A study on Co-authorship Network Based on Visualization Knowledge Mapping: Study for a Journal, Library and Information Service. 12 (2011) 109-113. In Chinese.

[17] Shan-min DING, Application of Social Network Analysis Method in Co-authorship Network, Tianjin: Tianjin Normal University, 2012. In Chinese.

[18]Zhi-hong LI, Qian MA, Guang-gang ZHOU, Analysis on the Time Evolution of Cross-University Academic Papers Co-author Network in the Filed of Management Science within China, Journal of Industrial Engineering and Engineering Management, 4 (2013) 126-136. In Chinese.

[19] Ji-tao GONG, Xiao-feng WEI, A Study on Patent Co-author Network Based on Social Network Analysis: Wind Power Technology Patents in China as an Example, Journal of Information. 11 (2013) 37-42. In Chinese.

[20] Xian-qian RONG, Tao TAN, An Empirical Study on Co-authorship Analysis in the Field of Library and Information Science with Social Network Analysis, Library World. 1 (2010) 1-5. In Chinese. 